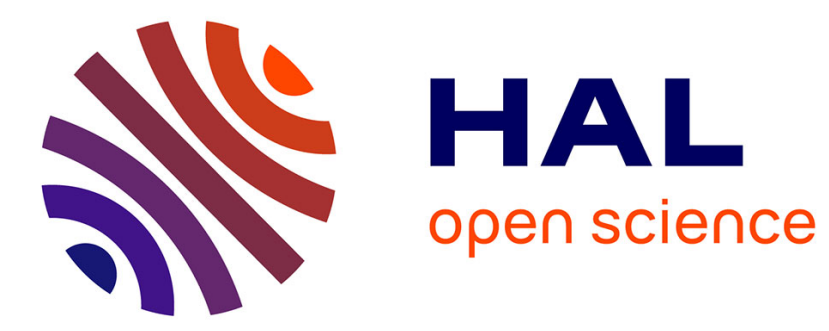

\title{
Angular and Spectral Bandwidth of Extreme UV Multilayers Near Spacer Material Absorption Edges
}

\author{
A A Zameshin, A E Yakshin, A Chandrasekaran, F. Bijkerk
}

\section{To cite this version:}

A A Zameshin, A E Yakshin, A Chandrasekaran, F. Bijkerk. Angular and Spectral Bandwidth of Extreme UV Multilayers Near Spacer Material Absorption Edges. Journal of Nanoscience and Nanotechnology, 2019, 19 (1), pp.602 - 608. 10.1166/jnn.2019.16478 . hal-01909422

\section{HAL Id: hal-01909422 \\ https://hal.science/hal-01909422}

Submitted on 20 Nov 2018

HAL is a multi-disciplinary open access archive for the deposit and dissemination of scientific research documents, whether they are published or not. The documents may come from teaching and research institutions in France or abroad, or from public or private research centers.
L'archive ouverte pluridisciplinaire HAL, est destinée au dépôt et à la diffusion de documents scientifiques de niveau recherche, publiés ou non, émanant des établissements d'enseignement et de recherche français ou étrangers, des laboratoires publics ou privés. 


\title{
Angular and Spectral Bandwidth of Extreme UV Multilayers Near Spacer Material Absorption Edges
}

\author{
A. A. Zameshin*, A. E. Yakshin, A. Chandrasekaran, and F. Bijkerk \\ Industrial Focus Group XUV Optics, MESA+ Institute for Nanotechnology, University of Twente, 7522 NB Enschede, The Netherlands
}

\begin{abstract}
High resolution imaging systems for EUV range are based on multilayer optics. Current generation of EUV lithography uses broadband Sn LPP sources, which requires broadband mirrors to fully utilize the source power. On the other hand, there always remains a possibility to use FEL or synchrotron as EUV source. FEL can produce very bright narrowband EUV light of a tunable wavelength, and the spectral bandwidth of the mirror is no longer a restriction. In this paper we look at the consequences of switching to different wavelengths if FEL source is used. For instance, it is known that the reflectance of Mo/Si multilayers increases when approaching Si L-edge, and the spectral bandwidth drops. But the behavior of an angular bandwidth (and its relation with the spectral bandwidth) is usually left out. It is also sometimes assumed that these bandwidths are correlated. For a large aperture EUV optical system with diffraction-limited resolution angular acceptance of a mirror is also a very important parameter. We show that the angular bandwidth of several multilayer systems (Mo/Si, Mo/Be, Ru/Si, Ru/B, La/B) actually increases close to spacer absorption edges, opposite to what occurs with the spectral bandwidth. We study this effect and show that it is caused by an interplay of changing optical constants of respective materials used in these multilayer combinations. We also provide an experimental check of the angular bandwidth of $\mathrm{Mo} / \mathrm{Si}$ multilayers at 13.5 and $12.6 \mathrm{~nm}$, which confirms our calculations.
\end{abstract}

Keywords: Mutlilayer, EUV Photolithography, Free Electron Laser.

\section{INTRODUCTION}

High resolution imaging systems for the Extreme UV (EUV) wavelength range are based on multilayer reflective optics. ${ }^{1}$ The most advanced imaging system in microelectronics technology to date is EUV photolithography at $13.5 \mathrm{~nm}$ wavelength, hence the motivation for our work lies in the possible improvement of $\mathrm{Mo} / \mathrm{Si}$-based multilayers. These mirrors have a theoretical reflectance of more than $73 \%$ (Si-capped) at near normal incidence, with the best experimentally produced interface engineered multilayers reaching a reflectance of $70.3 \% .^{2}$ Since the optical systems may consist of up to 11 mirrors, the total throughput is essentially reduced. This emphasizes the importance of maximizing the mirror reflectivity and, at the same time, the yield of the respective EUV light source. There are several different source types available in EUV range: ${ }^{3}$ laser produced plasmas (LPP), discharge produced plasmas (DPP), free electron lasers (FEL) and synchrotrons.

*Author to whom correspondence should be addressed.
The first two are more compact, and allow for a granular lithography approach, which may explain why they are currently considered as mainstream sources for EUV lithography. These sources can be broadband or narrowband, depending on the emission spectrum used. The prevailing high brightness source for industrial needs uses a broad $\mathrm{Sn}$ line spectrum around $13.5 \mathrm{~nm}$. To achieve higher throughput and higher integrated reflectance such broadband source needs broadband mirrors.

EUV multilayers are designed for a wavelength slightly above the absorption edge of the spacer material. For example, it is known ${ }^{4}$ that the maximal reflectance of a $\mathrm{Mo} / \mathrm{Si}$ multilayer mirror in EUV range depends on a wavelength - the closer to $\mathrm{Si}$ absorption edge, the higher the reflectance. It is also known that the spectral bandwidth of $\mathrm{Mo} / \mathrm{Si}$ multilayer mirror decreases when approaching the edge. Therefore for a broadband source, where the total throughput depends on the integrated reflectance over the given spectral range, the optimal wavelength would be away from the edge. ${ }^{4}$ Alternative light sources such as free electron lasers (FELs) are capable of producing a narrow 
spectrum of EUV light of very high average power (examples are $\mathrm{FLASH}^{5}$ and FERMI ${ }^{6}$ ). In the case of FEL the operational wavelength can be shifted close to Si L-edge in order to obtain more reflectance from the Mo/Si mirrors.

Although for FEL the spectral bandwidth of the mirror is no longer a restriction for an optical system, the angular bandwidth still might be. For a diffraction-limited optical system it influences the numerical aperture, and therefore affects resolution as well. It is generally assumed that the angular and spectral bandwidths are correlated. However as it will be shown here, it is not always the case. In this paper we first investigate an interplay between the angular and spectral bandwidth theoretically for several different multilayers, $\mathrm{Mo} / \mathrm{Si}, \mathrm{Mo} / \mathrm{Be}, \mathrm{Ru} / \mathrm{Si}, \mathrm{Ru} / \mathrm{B}$, and $\mathrm{La} / \mathrm{B}$. After that we perform an experimental check of the observed behavior on an example of a Mo/Si multilayer, considering the lack of multi-wavelength measurements of the angular dependencies of EUV multilayers in literature.

\section{THEORETICAL}

Following the Bragg's law $2 d \sin \theta_{0}=m \lambda_{0}$, it could be intuitively concluded that the angular and spectral bandwidths are correlated. This assumption is supported by the following analytical approximation: ${ }^{7}$

$$
\Delta \theta=2 \sqrt{\frac{\Delta \lambda}{\lambda_{0}}}
$$

where $\Delta \theta$ and $\Delta \lambda$ are the angular and spectral bandwidths (full width of half maximum of the corresponding Bragg peak, as shown in Fig. 1), and $\lambda_{0}$ is the resonance wavelength in the Bragg's equation. $\theta_{0}$-the resonance angle in the Bragg's equation, defined with respect to the sample surface-is equal to $90^{\circ}$. An underlying assumption made in the derivation of Eq. (1) is that the optical constants $(n=1-\delta+i \beta$, where $\delta$ and $\beta$ we also call optical constants) of elements composing a multilayer are not changing with wavelength. However, it is well known that even away from absorption edges optical constants are not constant. ${ }^{8}$ We decided to test the validity of Eq. (1) and calculate the behavior of the angular and spectral bandwidths for the realistic situation of varying optical constants.

The calculations of the multilayer reflectance were carried out using the Abeles matrix formalism in an algorithm described in Ref. [9]. All the theoretical dependencies presented in this paper are calculated for $s$-polarized light for the multilayers with period thicknesses and layer fractions ( $\Gamma$-ratios) optimized for maximal reflectance at a given wavelength and incidence angle. For simplicity we consider ideal multilayer structures with no roughness or interlayers. The number of bilayers in each case is always high enough that the residual transmittance through the multilayer stack is $\ll 1 \%$. The optical constants of given elements are calculated from the atomic scattering factors of the corresponding elements, taken from CXRO database. ${ }^{8}$

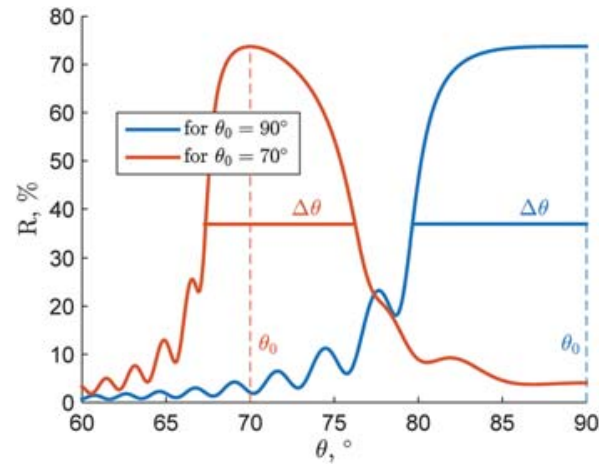

Figure 1. Reflectivity of ideal Mo/Si multilayers at $13.5 \mathrm{~nm}$ as a function of the incident angle. The multilayers are optimized for $\theta_{0}=90^{\circ}$ and $\theta_{0}=70^{\circ}$. The corresponding FWHM of the Bragg peaks $\Delta \theta$ are also shown.

We calculated wavelength dependencies of the maximal achievable reflectance $R_{\max }$, spectral and angular bandwidths $\Delta \lambda$ and $\Delta \theta$ for several different multilayer systems: $\mathrm{Mo} / \mathrm{Si}, \mathrm{Ru} / \mathrm{Si}, \mathrm{Mo} / \mathrm{Be}, \mathrm{Ru} / \mathrm{Be}, \mathrm{Mo} / \mathrm{Y}, \mathrm{Ru} / \mathrm{Y}, \mathrm{Ru} / \mathrm{B}, \mathrm{La} / \mathrm{B}$. To avoid redundant information we chose a representative selection of the key systems which illustrates well the generally observed behavior: $\mathrm{Mo} / \mathrm{Si}, \mathrm{Ru} / \mathrm{Si}, \mathrm{Mo} / \mathrm{Be}, \mathrm{Ru} / \mathrm{B}$, $\mathrm{La} / \mathrm{B} . \mathrm{Mo} / \mathrm{Si}$ is the key multilayer combination for EUV lithography, while $\mathrm{Ru} / \mathrm{Si}$ can be a viable wideband replacement of $\mathrm{Mo} / \mathrm{Si}$ in EUV photomasks, having lower penetration depth and higher spectral bandwidth. ${ }^{10,11} \mathrm{Mo} / \mathrm{Be}$ and $\mathrm{La} / \mathrm{B}$ are included as the optional choices of highreflectance multilayers for the wavelengths lower than $13.5 \mathrm{~nm}^{12,13}$ Only these selected multilayer combinations are used for the calculation presented in Figure 2. The presented dependencies $R=f\left(\lambda_{0}\right)$ are well known in literature, and their behavior is confirmed by many experiments carried away from the corresponding absorption edges of the spacers. The dependencies $\Delta \lambda=f\left(\lambda_{0}\right)$ in Figure 2(B) behave as expected as well: $\Delta \lambda$ scales with the wavelength $\lambda_{0}$. On the other hand, $\Delta \theta=f\left(\lambda_{0}\right)$ in Figure 2(C) have more complex behavior. To check applicability of the approximation (1) we plot $\Delta \theta$ as a function of $\sqrt{\Delta \lambda / \lambda_{0}}$ in Figure 2(D). The resulting dependence does not always follow the expected straight line. For each multilayer there is a region of $\lambda_{0}$ in which it behaves close to Eq. (1), but closer to the absorption edge of a spacer the dependence always strongly deviates from Eq. (1). Moreover, in this region, $\Delta \theta$ and $\Delta \lambda$ start to change in different directions, $\Delta \lambda$ decreases while $\Delta \theta$ increases. For B- and Be-based multilayers this effect is observed very close to the corresponding edge: $\sim 6.6-7.0 \mathrm{~nm}$ for $\mathrm{Ru} / \mathrm{B}, \sim 6.6-7.8 \mathrm{~nm}$ for $\mathrm{La} / \mathrm{B}$, and $11.2-11.8 \mathrm{~nm}$ for $\mathrm{Mo} / \mathrm{Be}$. For Si-based multilayers it is observed in a significantly wider range: from Si L-edge until $15.6 \mathrm{~nm}$ for $\mathrm{Mo} / \mathrm{Si}$ and $15.2 \mathrm{~nm}$ for $\mathrm{Ru} / \mathrm{Si}$. If in case of $\mathrm{B}$ and $\mathrm{Be}$ the proximity to the edge could still explain the deviation, it cannot for $\mathrm{Si}$. 

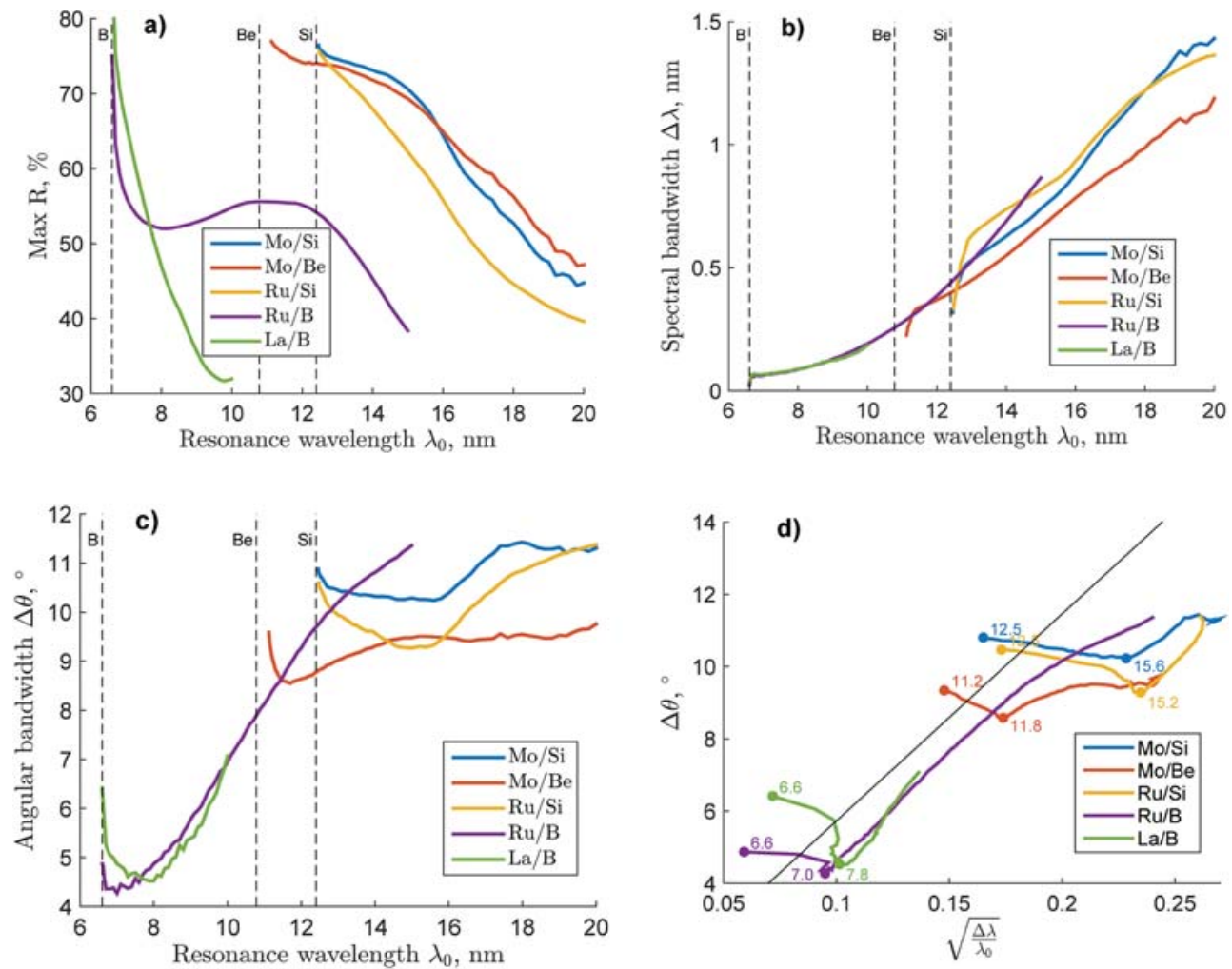

Figure 2. Calculated wavelength dependencies for several multilayers combinations, $\lambda_{0}$ from the spacer edge to $20 \mathrm{~nm}, \theta_{0}=90^{\circ}$ (normal incidence). The multilayer period and $\Gamma$-ratio are optimized for each point. La has its own edge at $92 \mathrm{eV}(\sim 13.5 \mathrm{~nm})$, therefore for La/B multilayers the wavelength range was limited to 6.6-10 nm. (a) $R=f\left(\lambda_{0}\right)$, generally increasing towards a spacer absorption edge. (b) $\Delta \lambda=f\left(\lambda_{0}\right)$, monotonously decreasing towards a spacer absorption edge. (c) $\Delta \theta=f\left(\lambda_{0}\right)$, showing non-monotonous behavior. (d) $\Delta \theta=f\left(\sqrt{\Delta \lambda / \lambda_{0}}\right)$, showing deviation from a straight line (black), expected by approximation (1). The deviation occurs closer to spacer edge, and especially pronounced for $\mathrm{Mo} / \mathrm{Si}$ and $\mathrm{Ru} / \mathrm{Si}$ from 12.5 to $15.6 \mathrm{~nm}$. For each curve two wavelengths (in $\mathrm{nm}$ ) are shown as solid dots: The point of the highest reflectance (closest to the absorption edge) and a turning point, at which $\Delta \theta=f\left(\sqrt{\Delta \lambda / \lambda_{0}}\right)$ changes the sign of its derivative.

The calculations shown above indicate that the approximation (1) cannot be applied to realistic systems with changing optical constants. On initial consideration the deviation from this approximation can be explained as follows. The spectral Bragg peak is influenced by the changes in the optical constants within the peak itself, while the angular Bragg peak is measured at a constant wavelength, therefore it is independent on the changes in the optical constants. However, this explanation can easily be proven wrong. It predicts that $\Delta \theta$ would not change its behavior. But in Figure 2 it is $\Delta \lambda$ that behaves monotonously, while $\Delta \theta$ is the parameter with more complex dependence.

To check the boundaries of approximation (1) we performed calculations of $R\left(\lambda_{0}\right), \Delta \lambda\left(\lambda_{0}\right)$ and $\Delta \theta\left(\lambda_{0}\right)$ for $\mathrm{Mo} / \mathrm{Si}$ multilayers, where optical constants of Mo Si or both were fixed to their corresponding values at $13.5 \mathrm{~nm}$. The relationship between $\Delta \theta$ and $\Delta \lambda$ is shown in Figure 3 . We observe that in case of the fixed optical constants for both $\mathrm{Mo}$ and $\mathrm{Si}, \Delta \theta$ monotonously decreases with $\Delta \lambda$ (Fig. 3, solid red curve). So in case of the $\mathrm{Mo} / \mathrm{Si}$ multilayer the approximation (1) will qualitatively comply if the underlying assumption of not changing optical constants with wavelength is fulfilled. At the next step we checked the conditions that would prevent the approximation from being complied. In particular we checked whether there is any specific optical constant of the spacer or reflector, which would be responsible for the broken correlation between the angular and spectral bandwidths. For that, we ran calculations with alternatively fixing each of the optical constants one at a time. The results shown in Figure 3 imply that only when all the optical constants are fixed at the same time, the bandwidth behavior is similar to the analytical approximation in the whole wavelength range. We could not achieve this by fixing any optical constant separately $\left(\delta_{\mathrm{Si}}, \beta_{\mathrm{Si}}, \delta_{\mathrm{Mo}}, \beta_{\mathrm{Mo}}\right)$ or in pairs $\left(\delta_{\mathrm{Si}}\right.$ and $\beta_{\mathrm{Si}}, \delta_{\mathrm{Mo}}$ and $\beta_{\mathrm{Mo}}$ ). As a result, we conclude that it is the changes in every single optical constant that are responsible for the deviation from the analytical approximation (1). The final optical response is determined by an interplay between all the optical constants of Mo and Si.

Additionally to the optical constants there is yet another quantity which should be playing a role in spectral and 


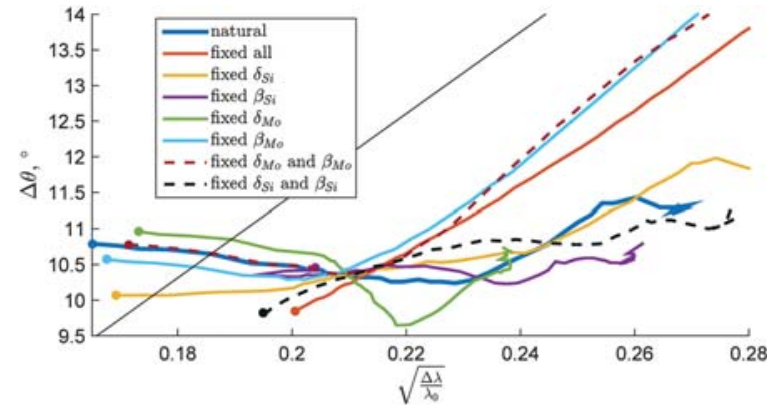

Figure 3. Calculated relation between $\Delta \theta$ versus $\Delta \lambda$ in a form of dependence $\Delta \theta=f\left(\sqrt{\Delta \lambda} / \lambda_{0}\right)$, shown for normal incidence ideal $\mathrm{Mo} / \mathrm{Si}$ multilayers with modified optical constants of Mo and Si. A straight line, expected by the approximation (1) is also shown. Only if all the optical constants $\left(\delta_{\mathrm{Si}}, \beta_{\mathrm{Si}}, \delta_{\mathrm{Mo}}, \beta_{\mathrm{Mo}}\right)$ are fixed, $\Delta \theta$ versus $\Delta \lambda$ relation resemble the approximation (1), although shifted.

angular bandwidth of a multilayer. It is penetration depth. However, since we show that the angular and spectral bandwidth are not necessarily correlated with each other, the penetration depth alone cannot be used to explain the different behavior of the angular and spectral bandwidth.

\section{EXPERIMENTAL DETAILS}

To perform an experimental comparison of the angular bandwidths of $\mathrm{Mo} / \mathrm{Si}$ multilayers at $13.5 \mathrm{~nm}$ and closer to $\mathrm{Si}$ edge, we deposited 50-period $\mathrm{Mo} / \mathrm{Si}$ multilayers, designed to reflect at near-normal incidence of $88.5^{\circ}$ at 13.5 and $12.6 \mathrm{~nm}$. We aimed for a relative comparison of these multilayers, there was no interface engineering or roughness control applied. The multilayers were deposited by DC magnetron sputtering at MS1600 coater designed by Roth and Rau, which has UHV base pressure $\left(10^{-8}\right.$ mbar). The coatings were done on superpolished $25 \times$ $25 \mathrm{~mm}^{2}$ Si substrates with native oxide and $0.15 \pm 0.05 \mathrm{~nm}$ surface roughness. The multilayers had an accurately controlled period thickness gradient along the surface of the samples to cover a certain range of wavelengths (at a fixed angle of incidence), or a range of angles of incidence (at a fixed wavelength) at which the multilayers would have the highest reflection. The samples designed for the wavelengths near $12.6 \mathrm{~nm}$ had period thicknesses in a range from 6.2 to $6.5 \mathrm{~nm}$, and the samples for near $13.5 \mathrm{~nm}$ had period thicknesses in a range from 6.7 to $7 \mathrm{~nm}$. The samples were $\Gamma$-optimized with a step of 0.05 in order to achieve the maximum reflectance at 13.5 and $12.6 \mathrm{~nm}$ at near-normal incidence.

EUV reflectance measurements of all of the samples were performed at Bessy II beam-line at PhysikalischTechnische Bundesanstalt (PTB) in Berlin. ${ }^{14}$ Each separate measurement essentially gives a spectral Bragg peak around a given wavelength (Fig. 4). Several points on each sample were measured, which allowed us to obtain experimental dependencies of $R\left(\lambda_{0}\right)$ and $\Delta \lambda\left(\lambda_{0}\right)$ for angle of

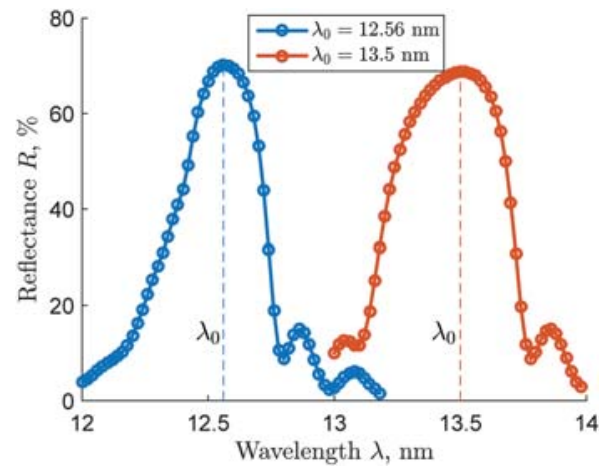

Figure 4. Spectral Bragg peaks of $\mathrm{Mo} / \mathrm{Si}$ multilayers, measured at $13.5 \mathrm{~nm}$ and $12.56 \mathrm{~nm}$.

incidence $1.5^{\circ}$ off-normal $\left(\theta_{0}=88.5^{\circ}\right)$. Note that EUV reflectance measurement setup at PTB can only measure up to $88.5^{\circ}$

It is less straightforward to measure $\Delta \theta\left(\lambda_{0}\right)$, so here it is described in more details. We selected several wavelengths $\lambda_{\text {sel }}$ at which $\Delta \theta$ would be measured. Then we selected the positions on the samples with the optimal period that would provide the highest reflectance at these given $\lambda_{\text {sel }}$ at near normal incidence, and measured $R(\theta)$ on them with a special procedure described below. For a fixed multilayer period the wavelength and incidence angle can still be varied, which allows to measure a number of $R(\theta)$ curves, which have maxima at different $\theta_{0}$. As shown in Figure 1, there can be a strong difference between these curves, as well as between the angular bandwidths $\Delta \theta$ extracted from them. A certain precision in determination of $\theta_{0}$ is required to enable a comparison of the angular bandwidths, so the measurement were done such that both $\theta$ and $\lambda$ were varied. This way the dependencies $\Delta \theta\left(\theta_{0}\right)$ for each $\lambda_{\text {sel }}$ were measured, with $\Delta \theta$ at $\theta_{0}=88.5^{\circ}$ being derived from these measurements.

The reflectance measurement setup at PTB can vary wavelength much more rapidly than the angle of incidence. Therefore in the measurement procedure we did not directly measure $R(\theta)$ curves for each $\theta_{0}$. Instead, we measured $R(\lambda)$ curves for each $\theta_{0}$. This effectively creates a three-dimensional matrix of triplets $(R, \theta, \lambda)$, therefore with enough density of points $\theta_{0}$ we can convert these dependencies into $R(\theta)$ for every $\lambda$. It is possible to obtain the desired form $R(\theta)$ for every $\theta_{0}$ as well. Each $R(\theta)$ has maximum $R$ at a point $\left(R_{\max }, \theta_{0}\right)$. This allows to sort dependencies $R(\theta)$ by $\theta_{0}$ instead of $\lambda$.

\section{RESULTS}

The results of all the measurements are shown in Figures 4 and 5. The Bragg peaks with the highest achieved reflectivity values for $13.5 \mathrm{~nm}$ and closest to the edge, $12.56 \mathrm{~nm}$, are presented in Figure 4. The experimental dependence $R\left(\lambda_{0}\right)$ is shown in Figure 5(A). Since our multilayer 

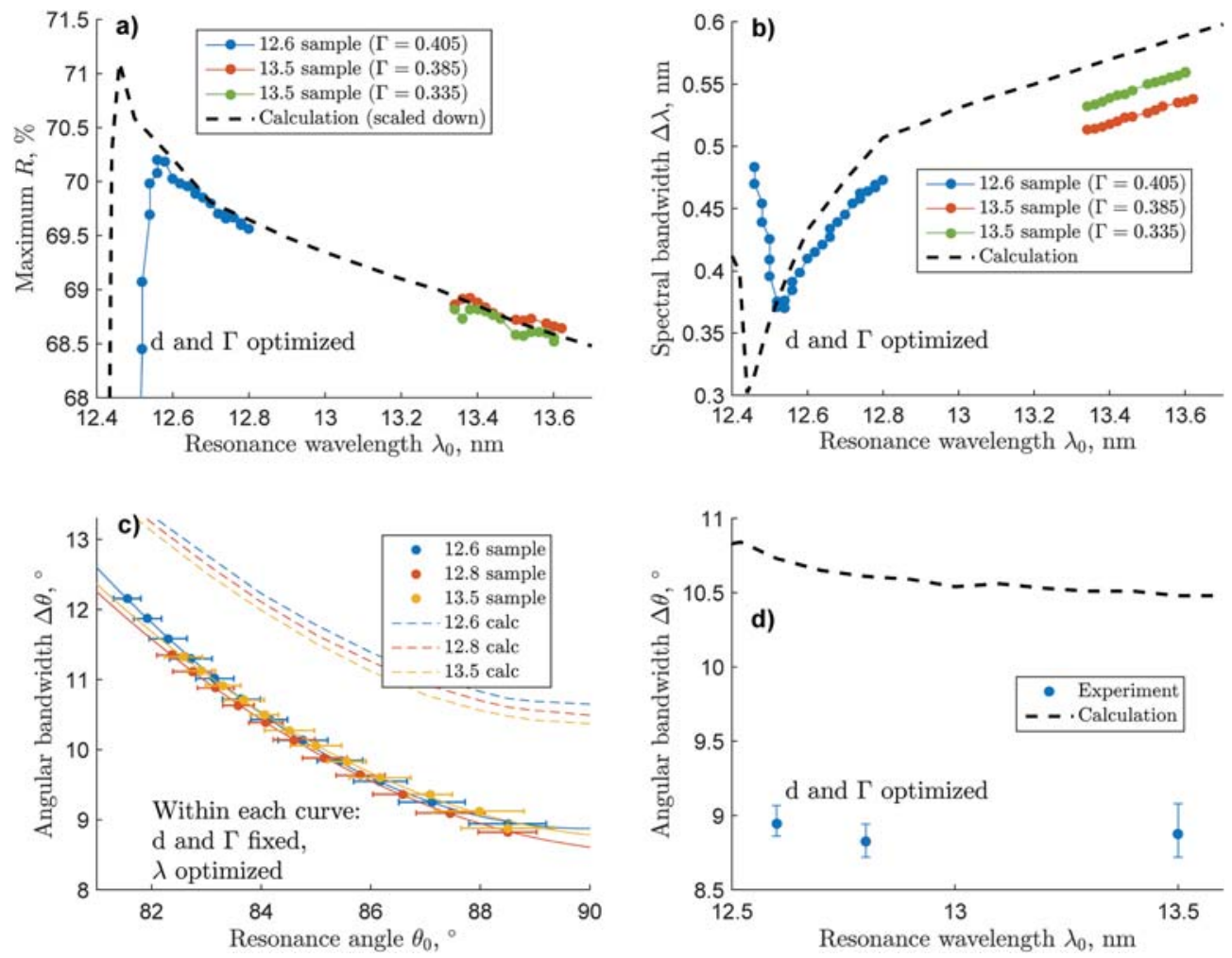

Figure 5. (a) Wavelength dependence of the highest reflectance of $\mathrm{Mo} / \mathrm{Si}$ multilayers at $\theta_{0}=88.5^{\circ}$ (experimental—solid points, theoretical—dashed line). The theoretical dependence was calculated for an ideal multilayer and scaled down for better visual comparison. The multilayer period $d$ and $\Gamma$-ratio were optimized. (b) Wavelength dependence of the spectral bandwidth $\Delta \lambda$ of $\mathrm{Mo} / \mathrm{Si}$ multilayers at $\theta_{0}=88.5^{\circ}$ (experimental—solid points, theoretical—dashed line). The multilayer period and $\Gamma$-ratio were optimized. (c) Dependences $\Delta \theta\left(\theta_{0}\right)$ (angular bandwidth as a function of resonance angle) for Mo/Si multilayers optimized for $13.5,12.8$ and $12.6 \mathrm{~nm}$. Within each curve the multilayer period and $\Gamma$-ratio were fixed, wavelength $\lambda$ was optimized to obtain the angular dependencies with maxima at different $\theta_{0}$. Experimental data-solid dots, connected with the best fitted 2nd degree polynomial, theoretical—dashed lines. (d) Wavelength dependence of the spectral bandwidth $\Delta \theta$ of $\mathrm{Mo} / \mathrm{Si}$ multilayers at $\theta_{0}=88.5^{\circ}$ (experimental—solid points, theoretical—dashed line). The multilayer period and $\Gamma$-ratio were optimized.

stacks were not interface engineered or optimized in terms of roughness, the maximum achieved reflectance was relatively low: $68.7 \%$ at $13.5 \mathrm{~nm}, 69.8$ at $12.7,70 \%$ at $12.6 \mathrm{~nm}$, and $70.2 \%$ at $12.56 \mathrm{~nm}$. Note that below $12.56 \mathrm{~nm}$ the reflectance starts to sharply decrease. The total uncertainty of each of these $R$ values is around $0.1 \%$ with a reproducibility of $0.05 \% .{ }^{15}$ The increase of $1.5 \%$ from 13.5 to $12.56 \mathrm{~nm}$ and $1.1 \%$ from 13.5 to $12.7 \mathrm{~nm}$ is similar to what was observed in other experiments: about $1 \%$ from 13.5 to 12.7 by Bajt et al., ${ }^{16}$ or Stuik et al. ${ }^{4}$ The obtained full experimental dependence $R\left(\lambda_{0}\right)$ has a trend very close to the calculated one (comparison shown in Fig. 5(A) with calculated curve scaled down), with only one notable exception - the drop of $R$ starts at a longer $\lambda_{0}$ in experiment than in theory. In experiment the highest possible reflectance is achieved at $12.56 \mathrm{~nm}$, while in calculations it is $0.1 \mathrm{~nm}$ lower.

The measurements of the spectral bandwidth $\Delta \lambda\left(\lambda_{0}\right)$ are presented in Figure 5(B). We observed a drop in $\Delta \lambda$ of $0.14 \mathrm{~nm}$, from $0.55 \mathrm{~nm}$ at $13.5 \mathrm{~nm}$ to $0.41 \mathrm{~nm}$ at $12.6 \mathrm{~nm}$. It corresponds well to the calculations, and so does the general behavior of $\Delta \lambda\left(\lambda_{0}\right)$ curve (comparison shown in Fig. 5(B)). $\Delta \lambda\left(\lambda_{0}\right)$ is much more sensitive to the exact $\Gamma$-ratio than $R\left(\lambda_{0}\right)$. However the onset for the abrupt jump of the spectral bandwidth occurs at a larger $\lambda_{0}$, just as in case of $R\left(\lambda_{0}\right)$.

The measurements of the angular bandwidths are shown in Figures 5(C) and (D). We selected three wavelength values at which this was measured: $\lambda_{\text {sel }}=12.6,12.8$ and $13.5 \mathrm{~nm}$. Figure 5(C) shows experimental and calculated dependencies $\Delta \theta\left(\theta_{0}\right)$ with extrapolation to $\theta_{0}=90^{\circ}$. All three curves $\Delta \theta\left(\theta_{0}\right)$ are indistinguishable within the presented error margins. Figure 5(D) shows the obtained values of $\Delta \theta$ at the highest measurable angle $\theta_{0}=88.5^{\circ}$ as a function of wavelength $\lambda_{0}$, and a comparison with the calculated dependence. For all three measured wavelengths $\Delta \theta$ turns out to be within the measurement uncertainties: $8.94 \pm 0.13^{\circ}$ for $12.6 \mathrm{~nm}, 8.81 \pm 0.12^{\circ}$ for $12.8 \mathrm{~nm}$, $8.9 \pm 0.2^{\circ}$ for $13.5 \mathrm{~nm}$. Therefore there is no experimentally observable trend. The calculated trend is quite small as well, and falls within the error margin of the experiment. So we do not observe a statistically significant 
increase in the angular bandwidth. But compared to the $25 \%$ drop of $\Delta \lambda$ we still show a significant deviation from the correlation between $\Delta \theta$ and $\Delta \lambda$, as expected from our calculations.

\section{DISCUSSION}

The highest measured reflectivity for $\mathrm{Mo} / \mathrm{Si}$ multilayer was achieved at $12.56 \mathrm{~nm}$, while the calculations using the atomic scattering factors from CXRO show the highest reflectivity at $12.45 \mathrm{~nm}$. This difference of $0.1 \mathrm{~nm}$ is significant and must be associated with the choice of the optical constants. For comparison, in an earlier work there was no noticeable difference in the position of maximum reflectance observed near the $\mathrm{B}$ absorption edge at $\sim 6.6 \mathrm{~nm}$ in for LaN/B multilayers, but for La/B multilayers the experimental position was $0.2 \mathrm{~nm}$ higher. ${ }^{17,18}$ In this work the data for $\mathrm{Si}$ were taken from the CXRO database for crystalline Si. However, the optical constants of the Si deposited in our work could differ. In addition to that, compound formation between Mo and Si can shift the position of the absorption edge. We were not able to find any good quality data for optical constants of molybdenum silicides close to the Si L-edge. However it is still possible to estimate a shift of the $\mathrm{Si}_{3}$-edge from the existing data about the binding energy shifts of $\mathrm{Si} 2 p^{3 / 2}$, using its XPS peak. This information became recently available for the full range of molybdenum silicides. ${ }^{19}$ However based on this data there is no defined direction in which the Si $2 p$ peak is expected to shift in $\mathrm{Mo} / \mathrm{Si}$ multilayers due to silicide formation. In a Si-rich compound such as $\mathrm{MoSi}_{2}$, the binding energy would shift to higher values up to $0.15 \mathrm{eV}$, while in a Mo-rich compound such as $\mathrm{Mo}_{5} \mathrm{Si}_{3}$ it would shift to lower values up to $0.15 \mathrm{eV}$. Different silicides are formed at different interfaces in $\mathrm{Mo} / \mathrm{Si}$ multilayers, with a tendency for $\mathrm{MoSi}_{2}$ formation at the Mo-on-Si interface, and $\mathrm{Mo}_{5} \mathrm{Si}_{3}$ at the Si-on-Mo interface. ${ }^{19}$ The Moon-Si interface is known to be thicker than the Si-on-Mo interface. ${ }^{20,21}$ On the other hand the silicide with lower Si $2 p$ energy $\left(\mathrm{Mo}_{5} \mathrm{Si}_{3}\right.$ at $\mathrm{Si}$-on-Mo interface) would play a bigger role at the high-wavelength side of the $\mathrm{Si}_{3^{-}}$ edge. It is difficult to predict how exactly this would influence the wavelength at which the reflectivity drop of the $\mathrm{Mo} / \mathrm{Si}$ multilayer is observed, but a simple estimation can be made. The shift of Si L-edge by $0.1 \mathrm{~nm}$ to the higher wavelengths would mean an energy shift of $0.85 \mathrm{eV}$. Such large energy shift is not expected from the available XPS data. So the silicide formation cannot explain the observed differences between the measured and calculated reflectivities near the Si absorption edge. Future studies will need to experimentally verify if the optical constants of the deposited $\mathrm{Si}$ can vary to the extent that explains the observed shift in Si L-edge.

It is difficult to accurately predict the effect of the angular bandwidth on an EUV imaging system, because it strongly depends on the design of that system. However, we can make a simple estimate for a photolithographic system in which the numerical aperture $N A \propto \sin \theta$. If the resolution of such a system is limited by diffraction and any other lithography process-related factors are constant, the minimal resolvable feature size (critical dimension) is $\mathrm{CD} \propto \lambda / N A$. We can use this formula to calculate the difference of $\mathrm{CD}$ when using a wavelength of 12.56 instead of $13.5 \mathrm{~nm}$. Based on the calculated $\Delta \theta$ values we can get a $11 \%$ decrease in $\mathrm{CD}$ when moving from 13.5 to $12.56 \mathrm{~nm}$. For the measured $\Delta \theta$ values we calculate a $7.9 \%$ decrease in $\mathrm{CD}$ when moving from 13.5 to $12.6 \mathrm{~nm}$. This allows us to conclude that, based on our calculations and the pilot experiment, there are potentially two advantages of using an EUV photolithographic system closer to the Si edge (at $\sim 12.56 \mathrm{~nm}$ ): a higher reflectivity (higher throughput) and a better resolution (lower $\mathrm{CD}$ ). In case of usage of a narrowband light source there are also no disadvantages, as the decrease of the spectral bandwidth is not a restriction for it.

\section{CONCLUSIONS}

We studied the wavelength dependence of the angular and spectral bandwidth of different multilayer reflective systems: $\mathrm{Mo} / \mathrm{Si}, \mathrm{Mo} / \mathrm{Be}, \mathrm{Ru} / \mathrm{Si}, \mathrm{Ru} / \mathrm{B}$ and $\mathrm{La} / \mathrm{B}$. According to an analytical approximation for constant optical constants, these bandwidths should be correlated. However, our calculations showed that in case of the changing optical constants with wavelength, as it occurs in real materials, the angular and spectral bandwidths are not always correlated. We demonstrate that the angular bandwidth of the considered multilayer systems actually increases towards spacer absorption edges, opposite to the spectral bandwidth. Based on the calculations we conclude that changes in any of the optical constants of the spacer or reflector result in the deviation from the analytical approximation. But it is an interplay between them that determines the final behavior of the optical response. The effect is especially significant in the vicinity of the absorption edges where the optical indices of materials change significantly.

The observed effect was checked experimentally on a $\mathrm{Mo} / \mathrm{Si}$ multilayer. The calculated and experimental trends of the reflectivity, angular and spectral bandwidths were found to be qualitatively in a good agreement. At the same time, the experimental values were systematically lower than the values calculated for ideal Mo/Si multilayers. Additionally it was found that the drop in reflectance associated with the Si absorption L-edge occurs at a higher wavelength than in calculations based on the atomic scattering factors from the CXRO database.

Both calculations and experimental data suggest that in case of a narrow band light source (e.g., a free electron laser) used for EUV imaging with $\mathrm{Mo} / \mathrm{Si}$ multilayer mirrors, the wavelength of choice should be closer to $\mathrm{Si}$ edge, around $12.56 \mathrm{~nm}$ (in this research), instead of the currently used $13.5 \mathrm{~nm}$. The obvious benefit is about a 
$1.5 \%$ higher reflectance per $\mathrm{Mo} / \mathrm{Si}$ multilayer mirror near the $\mathrm{Si}$ edge, which would result in a better throughput (about 25\% higher for 11-mirror system) and reduced thermal load of the total optical system. Additionally we show that, counterintuitively, the angular bandwidth of $\mathrm{Mo} / \mathrm{Si}$ multilayers does not decrease when moving from $13.5 \mathrm{~nm}$ to the Si edge as could be expected based on the behavior of the spectral bandwidth. This means that the numerical aperture of an imaging system will not be affected while the printable feature size can be reduced by $8-11 \%$ due to moving from $13.5 \mathrm{~nm}$ to $12.56 \mathrm{~nm}$. The effect of wavelength is different for different multilayers. For example, at $13.5 \mathrm{~nm} \mathrm{Ru} / \mathrm{Si}$ multilayers have a higher spectral bandwidth compared to $\mathrm{Mo} / \mathrm{Si}$ multilayers, but lower reflectance and angular bandwidth. At $12.56 \mathrm{~nm}$ the optical response of $\mathrm{Ru} / \mathrm{Si}$ becomes almost equivalent to $\mathrm{Mo} / \mathrm{Si}$, possibly giving additional freedom in the choice of materials.

Acknowledgments: We acknowledge the support of the Industrial Focus Group XUV Optics at the MESA+ Institute at the University of Twente, notably the industrial partners ASML, Carl Zeiss SMT AG, PANalytical, and TNO as well as the Province of Overijssel and NWO.

\section{References and Notes}

1. E. Spiller, Soft X-ray optics, SPIE-The International Society for Optical Engineering, Bellingham, Washington (1994).

2. J. Bosgra, E. Zoethout, A. M. J. Van Der Eerden, J. Verhoeven, R. W. E. Van De Kruijs, A. E. Yakshin, and F. Bijkerk, Appl. Opt. 51 (2012).

3. V. Banine and R. Moors, J. Phys. D. Appl. Phys. 37, 3207 (2004).

4. R. Stuik, E. Louis, A. E. Yakshin, P. C. Görts, E. L. G. Maas, F. Bijkerk, D. Schmitz, F. Scholze, G. Ulm, and M. Haidl, J. Vac. Sci. Technol. B Microelectron. Nanom. Struct. 17, 2998 (1999).

5. W. Ackermann, G. Asova, V. Ayvazyan, A. Azima, N. Baboi, J. Bähr, V. Balandin, B. Beutner, A. Brandt, A. Bolzmann, R. Brinkmann, O. I. Brovko, M. Castellano, P. Castro, L. Catani, E. Chiadroni, S. Choroba, A. Cianchi, J. T. Costello, D. Cubaynes, J. Dardis, W. Decking, H. Delsim-Hashemi, A. Delserieys, G. Di Pirro, M. Dohlus, S. Düsterer, A. Eckhardt, H. T. Edwards, B. Faatz, J. Feldhaus, K. Flöttmann, J. Frisch, L. Fröhlich, T. Garvey, U. Gensch, C. Gerth, M. Görler, N. Golubeva, H.-J. Grabosch, M. Grecki, O. Grimm, K. Hacker, U. Hahn, J. H. Han, K. Honkavaara, T. Hott, M. Hüning, Y. Ivanisenko, E. Jaeschke, W. Jalmuzna, T. Jezynski, R. Kammering, V. Katalev, K. Kavanagh, E. T. Kennedy, S. Khodyachykh, K. Klose, V. Kocharyan, M. Körfer, M. Kollewe, W. Koprek, S. Korepanov, D. Kostin, M. Krassilnikov, G. Kube, M. Kuhlmann, C. L. S. Lewis, L. Lilje, T. Limberg, D. Lipka, F. Löhl, H. Luna, M. Luong, M. Martins, M. Meyer, P. Michelato, V. Miltchev, W. D. Möller, L. Monaco, W. F. O. Müller, O. Napieralski, O. Napoly, P. Nicolosi, D. Nölle, T. Nuñez, A. Oppelt, C. Pagani, R. Paparella, N. Pchalek, J. Pedregosa-Gutierrez, B. Petersen, B. Petrosyan, G. Petrosyan, L. Petrosyan, J. Pflüger, E. Plönjes, L. Poletto, K. Pozniak, E. Prat, D. Proch, P. Pucyk, P. Radcliffe, H. Redlin,
K. Rehlich, M. Richter, M. Roehrs, J. Roensch, R. Romaniuk, M. Ross, J. Rossbach, V. Rybnikov, M. Sachwitz, E. L. Saldin, W. Sandner, H. Schlarb, B. Schmidt, M. Schmitz, P. Schmüser, J. R. Schneider, E. A. Schneidmiller, S. Schnepp, S. Schreiber, M. Seidel, D. Sertore, A. V. Shabunov, C. Simon, S. Simrock, E. Sombrowski, A. A. Sorokin, P. Spanknebel, R. Spesyvtsev, L. Staykov, B. Steffen, F. Stephan, F. Stulle, H. Thom, K. Tiedtke, M. Tischer, S. Toleikis, R. Treusch, D. Trines, I. Tsakov, E. Vogel, T. Weiland, H. Weise, M. Wellhöfer, M. Wendt, I. Will, A. Winter, K. Wittenburg, W. Wurth, P. Yeates, M. V. Yurkov, I. Zagorodnov, and K. Zapfe, Nat. Photonics 1, 336 (2007).

6. E. Allaria, R. Appio, L. Badano, W. A. Barletta, S. Bassanese, S. G. Biedron, A. Borga, E. Busetto, D. Castronovo, P. Cinquegrana, S. Cleva, D. Cocco, M. Cornacchia, P. Craievich, I. Cudin, G. D'Auria, M. D. Forno, M. B. Danailov, R. De Monte, G. De Ninno, P. Delgiusto, A. Demidovich, S. Di Mitri, B. Diviacco, A. Fabris, R. Fabris, W. Fawley, M. Ferianis, E. Ferrari, S. Ferry, L. Froehlich, P. Furlan, G. Gaio, F. Gelmetti, L. Giannessi, M. Giannini, R. Gobessi, R. Ivanov, E. Karantzoulis, M. Lonza, A. Lutman, B. Mahieu, M. Milloch, S. V. Milton, M. Musardo, I. Nikolov, S. Noe, F. Parmigiani, G. Penco, M. Petronio, L. Pivetta, M. Predonzani, F. Rossi, L. Rumiz, A. Salom, C. Scafuri, C. Serpico, P. Sigalotti, S. Spampinati, C. Spezzani, M. Svandrlik, C. Svetina, S. Tazzari, M. Trovo, R. Umer, A. Vascotto, M. Veronese, R. Visintini, M. Zaccaria, D. Zangrando, and M. Zangrando, Nat. Photonics 6, 699 (2012)

7. I. V. Kozhevnikov and A. V. Vinogradov, Journal of Russian Laser Research 16 (1995).

8. CXRO X-ray Database, http://henke.lbl.gov/optical_constants/at.

9. S. N. Yakunin, I. A. Makhotkin, K. V. Nikolaev, R. W. E. van de Kruijs, M. A. Chuev, and F. Bijkerk, Opt. Express 22, 20076 (2014)

10. O. Wood, S. Raghunathan, P. Mangat, V. Philipsen, V. Luong, P. Kearney, E. Verduijn, A. Kumar, S. Patil, C. Laubis, V. Soltwisch, and F. Scholze, Proc. SPIE 9422, 94220I (2015).

11. O. R. Wood, K. Wong, V. Parks, P. A. Kearney, J. Meyer-Ilse, V. Luong, V. Philipsen, M. Faheem, Y. Liang, A. Kumar, E. Chen, C. Bennett, B. Fu, M. Gribelyuk, P. J. Mangat, P. Van der Heide, and W. Zhao, Proc. SPIE 9776, 977619 (2016).

12. E. Louis, A. E. Yakshin, T. Tsarfati, and F. Bijkerk, Prog. Surf. Sci. 86, 255 (2011)

13. D. S. Kuznetsov, A. E. Yakshin, J. M. Sturm, R. W. E. van de Kruijs, E. Louis, and F. Bijkerk, Opt. Lett. 40, 3778 (2015).

14. R. Klein, C. Laubis, R. Müller, F. Scholze, and G. Ulm, Microelectron. Eng. 83, 707 (2006)

15. C. Laubis, F. Scholze, C. Buchholz, A. Fischer, S. Hesse, A. Kampe, J. Puls, C. Stadelhoff, and G. Ulm, High-accuracy EUV reflectometry at large optical components and oblique incidence (2009), p. 72713 Y.

16. S. Bajt, J. B. Alameda, T. W. J. Barbee, W. M. Clift, J. A. Folta, B. Kaufmann, and E. A. Spiller, Opt. Eng. 41, 1797 (2002).

17. I. A. Makhotkin, E. Zoethout, E. Louis, A. M. Yakunin, S. Müllender, and F. Bijkerk, J. Micro/Nanolithography, MEMS, MOEMS 11, 40501 (2012).

18. I. A. Makhotkin, E. Zoethout, E. Louis, A. M. Yakunin, S. Müllender, and F. Bijkerk, Opt. Express 20, 11778 (2012).

19. E. Zoethout, E. Louis, and F. Bijkerk, J. Appl. Phys. 120, 115303 (2016).

20. S. Braun, H. Mai, M. Moss, R. Scholz, and A. Leson, Jpn. J. Appl. Phys. 41, 4074 (2002).

21. I. Nedelcu, R. W. E. van de Kruijs, A. E. Yakshin, and F. Bijkerk, J. Appl. Phys. 103, 83549 (2008). 